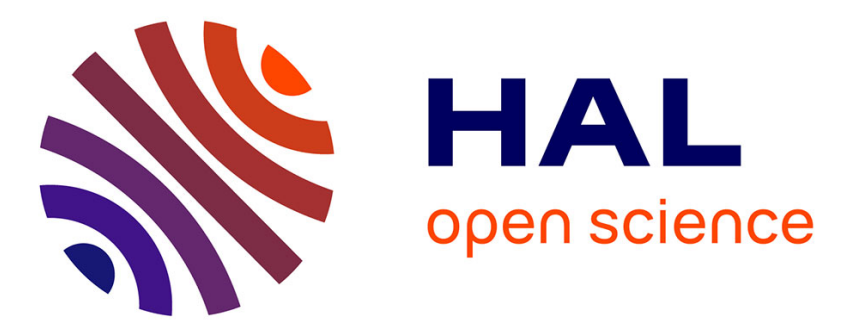

\title{
Analysis of flow induced by temperature fields in ratchet-like microchannels by Direct Simulation Monte Carlo
}

\author{
Jie Chen, Stefan K Stefanov, Lucien Baldas, Stéphane Colin
}

\section{To cite this version:}

Jie Chen, Stefan K Stefanov, Lucien Baldas, Stéphane Colin. Analysis of flow induced by temperature fields in ratchet-like microchannels by Direct Simulation Monte Carlo. International Journal of Heat and Mass Transfer, 2016, 99, pp.672-680. 10.1016/j.ijheatmasstransfer.2016.04.023 . hal-01877853v2

\section{HAL Id: hal-01877853 \\ https://hal.science/hal-01877853v2}

Submitted on 5 Apr 2019

HAL is a multi-disciplinary open access archive for the deposit and dissemination of scientific research documents, whether they are published or not. The documents may come from teaching and research institutions in France or abroad, or from public or private research centers.
L'archive ouverte pluridisciplinaire HAL, est destinée au dépôt et à la diffusion de documents scientifiques de niveau recherche, publiés ou non, émanant des établissements d'enseignement et de recherche français ou étrangers, des laboratoires publics ou privés.

\section{(ㄷ)(1) $\$$}

Distributed under a Creative Commons Attribution - NonCommerciall 4.0 International 


\author{
Analysis of flow induced by temperature fields in ratchet-like \\ microchannels by Direct Monte Carlo Simulation \\ Jie Chen ${ }^{1}$, Stefan K. Stefanov ${ }^{2}$, Lucien Baldas ${ }^{1}$, Stéphane Colin*1 \\ ${ }^{1}$ Institut Clément Ader (ICA), Université de Toulouse, CNRS-INSA-ISAE-Mines Albi-UPS, Toulouse \\ France \\ ${ }^{2}$ Institute of Mechanics - BAS, Acad. G. Bontchev St., bl. 4, 1113 Sofia, Bulgaria \\ *Corresponding author: stephane.colin@insa-toulouse.fr
}

\begin{abstract}
This paper presents a numerical analysis of a novel configuration of Knudsen pump for rarefied gas flow. The pumping element consists of two facing isothermal ratchet surfaces at different temperatures. The asymmetric saw-tooth-like surfaces in optimal geometrical conditions can create temperature inequalities in the near wall region and finally engender a macroscopic flow. The rarefied gas flow is numerically investigated by Direct Simulation Monte Carlo (DSMC) in the slip and transition regimes. A parametric study reveals the effects of several crucial parameters including temperature difference, Knudsen number, accommodation coefficients as well as main geometrical parameters. In particular, the results indicate that the mass flow rate reaches a maximum value for a Knudsen number around 0.1 and becomes negative for a Knudsen number close to unity.
\end{abstract}

\title{
Keywords
}

Thermally driven flow; rarefied gas; ratchet; Knudsen micropump; DSMC 


\section{Nomenclature}

\section{Symbols}

$c_{r} \quad$ relative velocity of colliding molecules

$d \quad$ ratchet depth

$F_{N} \quad$ number of real molecules represented by a test molecule

$h \quad$ distance between the planes of the ratchet tips

$h_{0} \quad$ smallest distance between two walls in the $y$-direction

Kn Knudsen number

$L \quad$ characteristic length, ratchet pattern length

$L_{m} \quad$ misalignment distance between ratchet patterns of facing walls

$N \quad$ number of test molecules

$N_{C} \quad$ maximum collision number

$n \quad$ normal coordinate

$P \quad$ pressure

$T \quad$ temperature

$t \quad$ time

$s \quad$ tangential coordinate

$u \quad$ velocity in $x$-direction

$v \quad$ velocity in $y$-direction

$V_{C} \quad$ cell volume

$x \quad$ Cartesian coordinate (streamwise direction)

$y \quad$ Cartesian coordinate (spanwise direction)

\section{Greek symbols}

$\alpha \quad$ tangential momentum accommodation coefficient

$\beta \quad$ ratchet angle

$\lambda$ molecular mean free path

$\mu \quad$ dynamic viscosity

$\sigma_{T} \quad$ collision cross section

Subscript

$\begin{array}{ll}h & \text { hot wall } \\ c & \text { cold wall } \\ V & \text { vertical wall } \\ I & \text { inclined wall }\end{array}$

\section{Introduction}


In rarefied gases, flows can be generated by a tangential temperature gradient applied along a wall without any initial pressure gradient. This well-known phenomenon is the socalled thermal transpiration (or thermal creep) effect. It is the basis of the Knudsen pump operation principle, which allows gas pumping without any moving part when the gas is under rarefied conditions. This kind of pump could provide reliable and precise control of gas microflows for a variety of applications, such as gas-sensing breath analyzers, chemical weapons detectors or micro vacuum generators directly integrated in MEMS. Following the seminal work of Knudsen [1], several different designs of Knudsen pumps have been studied in the past years. The typical Knudsen micropump is based on a cascade system in which a basic unit is composed of a microchannel [2-4] or a microporous medium [5, 6] connecting two mini chambers with different temperatures. An alternative configuration has been proposed and numerically studied, consisting of alternately connected curved and straight microchannels [7-9] or curved microchannels with different curvature radii [10]. A thermal edge compressor has been devised and experimentally investigated by Sugimoto [11]. This compressor includes an array of unheated plates made in ceramic that are facing an array of heated plates made of a Nichrome ribbon heater.

A new promising configuration of Knudsen pump consisting of two facing isothermal surfaces at different temperatures, one of which at least being unsymmetrically nanostructured, has recently been proposed and studied [12-14]. This so-called "ratchet micropump" is composed of isothermal surfaces at two different temperatures. The major advantage of this type of Knudsen pump is that the temperature gradients within the gas along the walls result from the specific structuration of isothermal walls rather than from a nonuniform temperature distribution along the solid walls. Consequently, the fabrication and temperature control of a ratchet pump are much easier than those of conventional Knudsen pumps, which require a periodic temperature distribution with temperature gradients along the 
microchannels and opposed temperature gradients in mini chambers. In all cases, heated and cooled zones of very small areas are usually required to achieve the appropriate periodic temperature distribution. The influence of the area of these heated and cooled zones has been numerically investigated for a micropump based on alternately connected curved and straight micro-channels [9] and practical limitations have also been described. Moreover, the control of temperature becomes more complicated when more stages are used to create a more powerful pump. In contrast, the heating/cooling system of a ratchet pump is relatively convenient to design. A multi-stage ratchet pump with increased power could be easily manufactured by simply using surfaces with several ratchet elements, without adding issues linked to temperature control.

In the original ratchet pump configuration, several special surface boundary conditions have been assumed. For example, a structured surface combining diffusive and specular segments was considered by Donkov et al. [12], who analysed a two-dimensional prototype channel which is composed of a cold flat wall and a hot ratchet surface (Fig. 1a). The cold flat wall is parallel to the $x$-direction and has a diffusive surface, whereas the hot ratchetstructured wall exhibits a periodic geometrical pattern divided into three segments: two diffusive boundary segments in the $x$ - and $y$-directions linked by one tilted specular segment. Donkov et al. demonstrated that: (i) a net flow rate is thermally generated in the $x$-direction when the gas is rarefied; (ii) there is no momentum in the $x$-direction when the entire structured wall has a diffuse surface and the asymmetry of the wall topography is not a sufficient condition to generate a flow along the channel. Würger proposed a ratchet pump design [13] based on the same physical mechanism as mentioned in [12]. It consists of two facing ratchet surfaces with the same topography maintained at a respectively hot and cold uniform temperature (Fig. 1b). Würger developed a simplified analytical model assuming a small ratchet depth $d$ (i.e. valid for $d<h$, where $h$ is the distance between the two planes of 
the ratchet tips). It was demonstrated that velocities of several meters per second could be obtained and that the thermally driven flow phenomenon should also be observed for a larger distance between both plates, up to hundred times higher than the mean free path of the molecules. However, the thermal creep along the vertical wall segments was neglected in Würger's model.

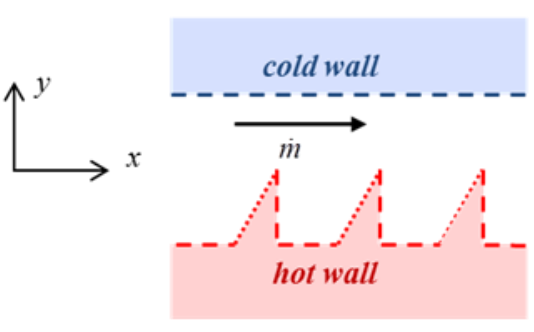

(a)

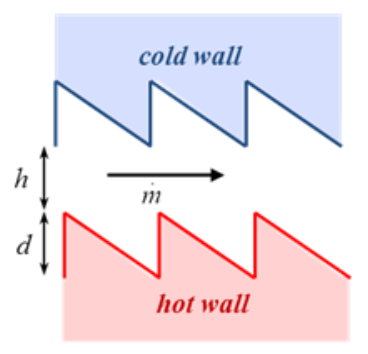

(b)

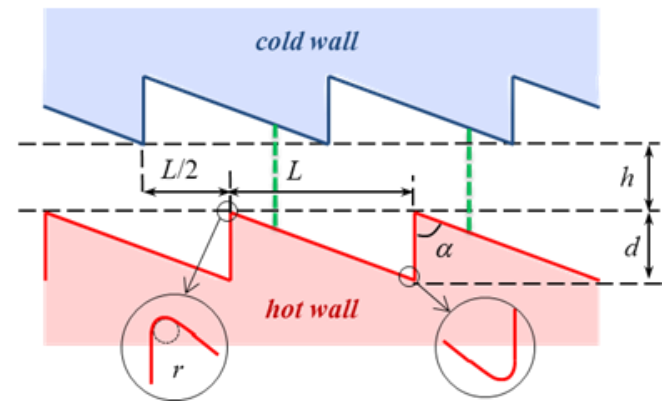

(c)

Fig. 1: Three ratchet designs analyzed by

(a) Donkov et al. [12], (b) Würger [13] and (c) Chen et al. [14].

In (a), diffusive walls are represented by dashed lines and specular walls by dotted lines.

Recently, by means of a Computational Fluid Dynamics (CFD) approach, three of the present authors numerically studied the performance of a new type of Knudsen pump with a misalignment of the upper and lower ratchet patterns (Fig. 1c) [14]. Using the Navier-Stokes equations with appropriate velocity slip and temperature jump boundary conditions, it was demonstrated that the pumping effect could be observed in the slip flow regime. Furthermore, the simulations revealed that the pump could operate even if all segments of the ratchet surfaces are diffusive walls. In other words, the finding emphasized the fact that a working device does not necessarily require, as claimed in [12], the presence of some specular segments, which are not practically achievable, as confirmed by most experimental measurements showing that the tangential accommodation coefficients range in practice from 0.85 to unity [15], which corresponds to rather diffusive wall conditions. Additionally, the influence of the geometric parameters and rarefaction was studied in [14]. It was observed 
that the mass flow rate increases with the Knudsen number in the rarefaction range corresponding to the slip flow regime. For the highest investigated values of the Knudsen number, the mass flow rate becomes less dependent of the Knudsen number, suggesting that a maximum value is expected in the early transition regime, where the Navier-Stokes based approach is not appropriate any longer.

In this paper, the new type of ratchet pump mentioned in [14], which consists of two hot and cold ratchet walls with misaligned identical saw-tooth patterns (Fig. 1c), is investigated by the Direct Simulation Monte Carlo (DSMC) method in a wide range of the Knudsen number covering both transition and slip flow regimes. The problem statement and numerical method are described in Section 2. Results on parametric studies and discussions are then presented in Section 3 and conclusions are drawn in Section 4.

\section{Problem statement and numerical method}

The studied configuration which consists of two hot and cold ratchet walls with misaligned identical saw-tooth patterns is shown in Fig. 2. The ratchet surfaces have a periodic pattern with a length $L$ in the $x$-direction and a height $d$ in the $y$-direction, resulting in an angle $\beta=\arctan (L / d)$. Due to the periodicity of the channel pattern, only a length $L$ of the channel is simulated in the present work. Periodic boundary conditions are implemented at the inlet and outlet, the positions of which are indicated by the green dot-dashed lines in Fig. 2 . The distance $h$ between the walls is defined from the planes of the ratchet tips. The misalignment distance in the $x$-direction between the two facing patterns is defined by the length $L_{m}$. The distance between the upper and lower walls is not constant and the smallest distance between the two walls in the $y$-direction is defined by the height $h_{0}$. The value of $h_{0}$ depends on the misalignment distance according to the relationship

$$
h_{0}=h+\left(1-L_{m} / L\right) d
$$


where $0<L_{m} \leq L$.

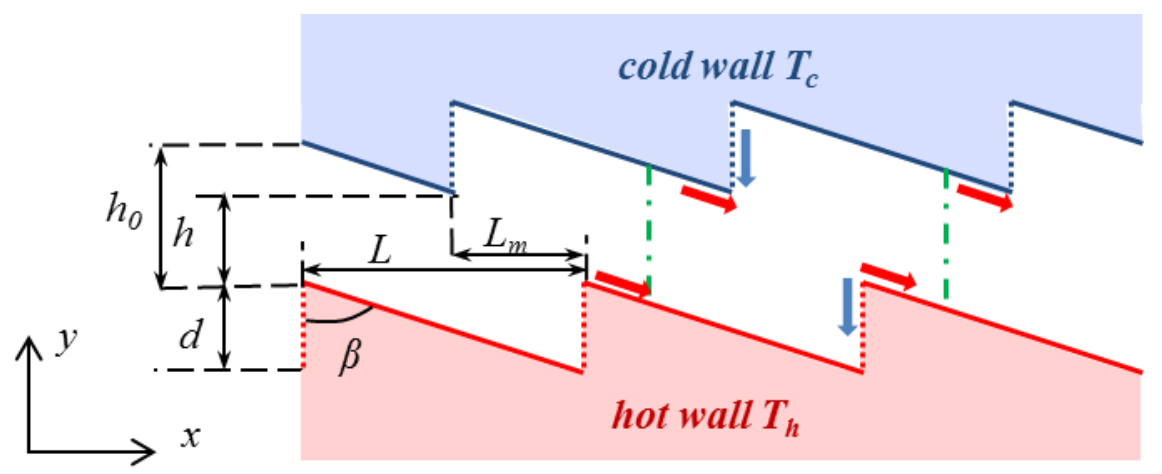

Fig. 2: Configuration and geometric parameters

The geometric configuration is close to the third configuration studied by CFD in [14] but, in order to simplify mesh generation and reduce computational cost, a sharp angle at the intersection of the segments is adopted in the present study instead of the tip radius introduced in [14] to take into account realistic micro fabrication constraints.

The Knudsen number

$$
K n=\sqrt{\frac{\pi}{2}} \frac{\mu_{0} \sqrt{R T_{0}}}{h P}
$$

is defined as the ratio of the mean free path $\lambda$ based on the Hard Sphere (HS) model over the height $h$. In Eq. (2), $P$ is the pressure and $R$ is the specific gas constant. The dynamic viscosity $\mu_{0}$ is defined at the mean temperature $T_{0}=\left(T_{c}+T_{h}\right) / 2$.

The DSMC method models the gas flow using a set of test molecules (simulators), each of them representing a large number of real molecules. Simulators move and collide in a probabilistic simulation in accordance with the rarefied gas dynamics theory. A fundamental assumption of the DSMC method is that the real process of molecular free movement and inter-molecular collisions can be approximated by decoupling it into two consecutive steps over time intervals smaller than the mean collision time. 
Based on the standard DSMC procedure [16, 17], a multi-block DSMC code with bodyfitted mesh has been developed to simulate the gas flow, subdividing the ratchet configuration into several computational domains with a simpler geometry. Previously, a mono-block version of the DSMC code has been used to investigate a rarefied gas flow in a rectangular enclosure induced by non-isothermal walls [18] and to analyze the influence of transversal walls on the stationary near-isothermal gas flow [19]. In this paper, a multi-block mesh is adopted to simplify the grid generation. For the studied configurations, the whole calculation domain is divided into three subdomains. Each subdomain is discretized by using uniform quadrilateral cells and the grids lines follow the boundaries of the domain.

A Hard Sphere (HS) model and the No Time Counter (NTC) scheme suggested by Bird [17] are implemented in the collision algorithm. A slight modification is introduced in the calculation of the maximum number of collisions $N_{C}$ in each cell [20], which is calculated according to

$$
N_{C}=\frac{1}{2} \frac{N(N-1) F_{N}\left(\sigma_{T} c_{r}\right)_{\max } \Delta t}{V_{C}}
$$

where $N$ is the actual number of test molecules in a cell, $F_{N}$ is the number of real molecules represented by a simulator, $\left(\sigma_{T} c_{r}\right)_{\max }$ is the maximum value of the product of the collision cross section and the relative velocity of the colliding molecules, $\Delta t$ is the time step and $V_{C}$ is the volume of the cell.

The macroscopic quantities are calculated by averaging the microscopic values of the test molecules on a given sampling cell over a large number of time steps. The sampling cell includes 4 collision cells, in each of which about 40 test molecules are arranged in the present simulations. Therefore the sampling size is defined as the product of the number of test molecules per cell, the number of collision cells in a sampling cell and the number of time steps during which the sampling is performed. Due to the very low macroscopic velocity of 
the gas flow, the simulations are carried out with a large sampling size of $10^{8}$. With this sampling size, the estimated error (associated with the one-standard-deviation error, based on the inverse square root of the sample size) on the macroscopic parameters is less than $1 \%$.

Regarding boundary conditions, each surface is assumed isothermal, which can be achieved in practice by materials of high thermal conductivity. The temperature of the lower hot wall is denoted by $T_{h}$ and the temperature of the upper cold wall is denoted by $T_{c}$. The well-known Maxwell's specular-diffuse model is applied, according to which a fraction $(1-\alpha)$ of the molecules is reflected specularly while the remaining fraction $\alpha$ is reflected diffusely in accordance with the Maxwellian velocity distribution. This dimensionless coefficient $\alpha$ is the so-called accommodation coefficient, as the Maxwell boundary condition does not distinguish the tangential momentum and energy accommodation coefficients [21]. The accommodation coefficients for vertical walls and inclined walls are denoted by $\alpha_{V}$ and $\alpha_{I}$, respectively.

A reference configuration is considered for further discussion. In this reference configuration, the geometric parameters of the ratchet are $L=4 \mu \mathrm{m}, h=d=1 \mu \mathrm{m}$ and $L_{m}=L / 2$. The reference temperatures of the hot and cold walls are $T_{h}=375 \mathrm{~K}$ and $T_{c}=225 \mathrm{~K}$, respectively. A completely diffuse reflection boundary condition is considered for all walls in the reference configuration, i.e. $\alpha_{V}=\alpha_{I}=1$.

Since two-dimensional simulations are implemented in this work, a reasonable width of $20 \mu \mathrm{m}$ in the $z$-direction is considered when the mass flow rates are calculated. The influence of the temperature difference, the Knudsen number, the accommodation coefficients and geometric parameters, i.e. the ratchet angle $\beta$ and the misalignment distance $L_{m}$, is analyzed in the following section. In all following simulations, the gas is Argon. For DSMC 
simulations, the diameter of the molecules is considered as constant and is calculated from Bird's data [17] at the reference temperature.

\section{Results and discussion}

\subsection{Temperature distribution and flow characteristics}

As a consequence of the geometry and boundary conditions, the dominating temperature gradient is perpendicular to the surfaces, in contrast with conventional thermal creep flows generated by an imposed tangential temperature gradient along the walls. This results in a stronger tangential temperature gradient in the gas along the vertical walls than along the inclined walls of the ratchet surfaces. Figure 3 shows that the tangential temperature gradients along the vertical walls is two or three times higher than along the inclined walls for $K n=0.23$. In addition, it is found that strong temperature gradients appear near the tips of the ratchet and decrease sharply getting away from the tips. Therefore the main thermally-driven flow is generated near the tips as shown by arrows. The red arrows (Fig. 3a) show flows generated by the inclined walls (solid lines) and the blue ones (Fig. 3b) correspond to the flows due to vertical walls (dotted lines). The general trend and the order of magnitude of the temperature gradient along the inclined wall are comparable with the analytical results provided by Eq. (6) in ref [13] and a mean flow in the $x$-direction is expected from Fig. 3. The temperature gradient along the vertical wall is omitted in the simplified analytical model of Würger; however, and particularly around the tips of the ratchet, the temperature varies more rapidly along the vertical segments than along the inclined segments. As a consequence, the flow generated by the vertical walls, shown by the blue arrows, is stronger than the flow due to the inclined walls. It results in a two-dimensional flow rather than in a uniform flow in $x$ direction, as predicted in [13]. 


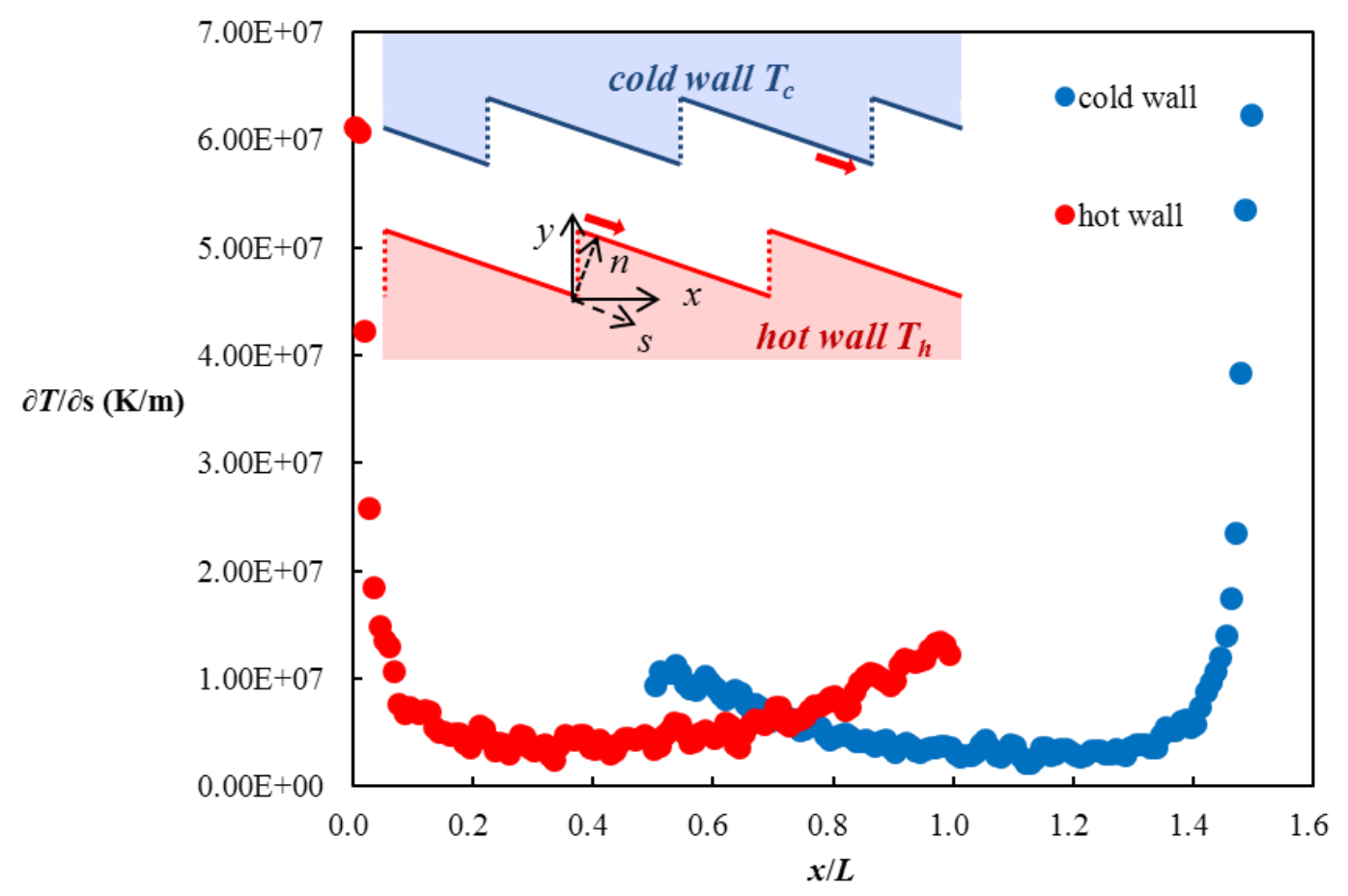

(a)

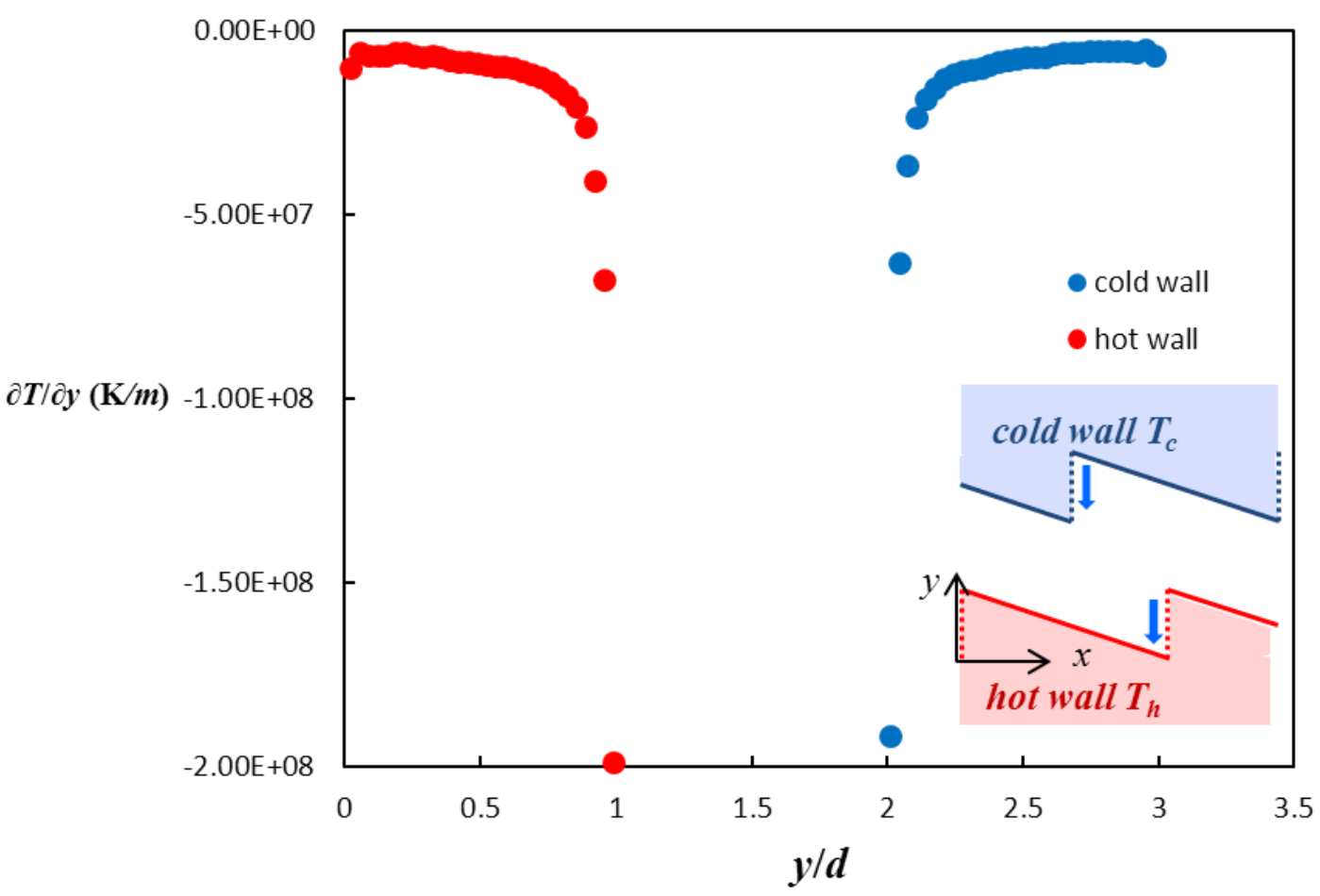

(b)

Fig. 3: Tangential temperature gradients in the gas close to the ratchet surfaces for the reference case at $K n=0.23$ along the a) inclined walls, b) vertical walls 
As a consequence, the actual flow field is a result of the combination of these local thermally-driven flows. Figure 4 presents the velocity vectors and the iso-contours of velocity magnitude. White arrows are added to clearly represent the global flow structure.

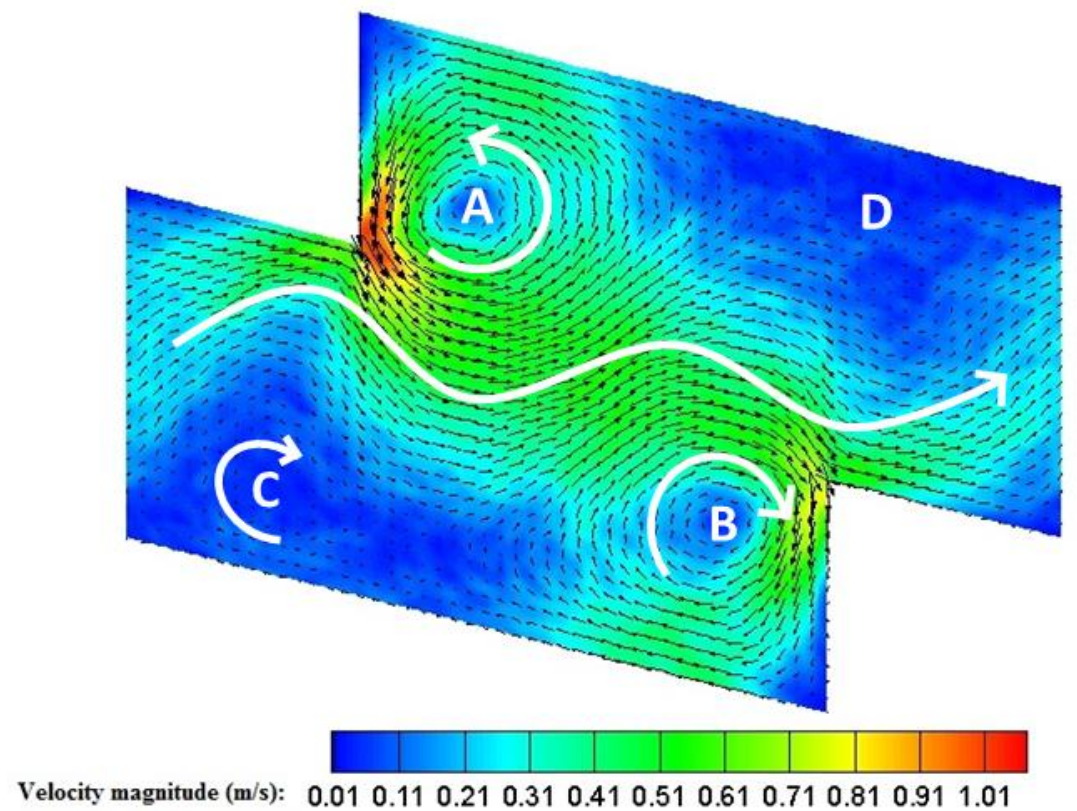

Fig. 4: Velocity vectors and iso-contours of velocity magnitude for the reference case at $K n=0.23$.

As shown in Fig. 4, the flow induced by the temperature difference between the two ratchet surfaces is much more complex than in a simple classic thermal creep flow between parallel plates. The main component of the temperature gradient is in the vertical direction from the upper cold region toward the lower hot region. This gradient within the fluid along the vertical walls generates a thermal creep flow from top to bottom, and a lower temperature gradient along the inclined walls generates a less intense thermal creep flow from left to right. The competition between the stronger creep flow along the vertical walls compared to the creep flow along the inclined walls results in an anticlockwise vortex $\mathrm{A}$ in the upper corners and in a clockwise vortex B in the lower corners. These two main counter-rotating vortices drag the gas along a serpentine path from left to right, generating a net flow in the $x$-direction, 
as shown by the white arrow in Fig. 4. This net flow has a stronger effect than the weak thermal creep flow along the lower inclined wall and is able to generate a low intensity secondary clockwise vortex $\mathrm{C}$. In addition, the net flow in the $x$-direction contributes to a slight stretching of vortex A and compression of vortex B, limiting the possible development of a secondary vortex in region D. This effect is more visible in Figs. 8a to 8c when the net flow increases with the Knudsen number.

The temperature difference between the two surfaces is the main factor controlling this thermally-driven flow. Thus, the influence of the temperature difference $\Delta T$ is studied by changing the temperature of the two surfaces while maintaining a constant mean temperature $T_{0}=300 \mathrm{~K}$. Figure 5 shows that the mass flow rate linearly increases with the temperature difference $\Delta T$. Even for a small temperature difference $\Delta T=50 \mathrm{~K}$, a significant mass flow rate of $7.69 \times 10^{-13} \mathrm{~kg} \mathrm{~s}^{-1}$ is obtained, which is measurable with a good accuracy for pressure driven [22] as well as for temperature driven gas microflows [23, 24].

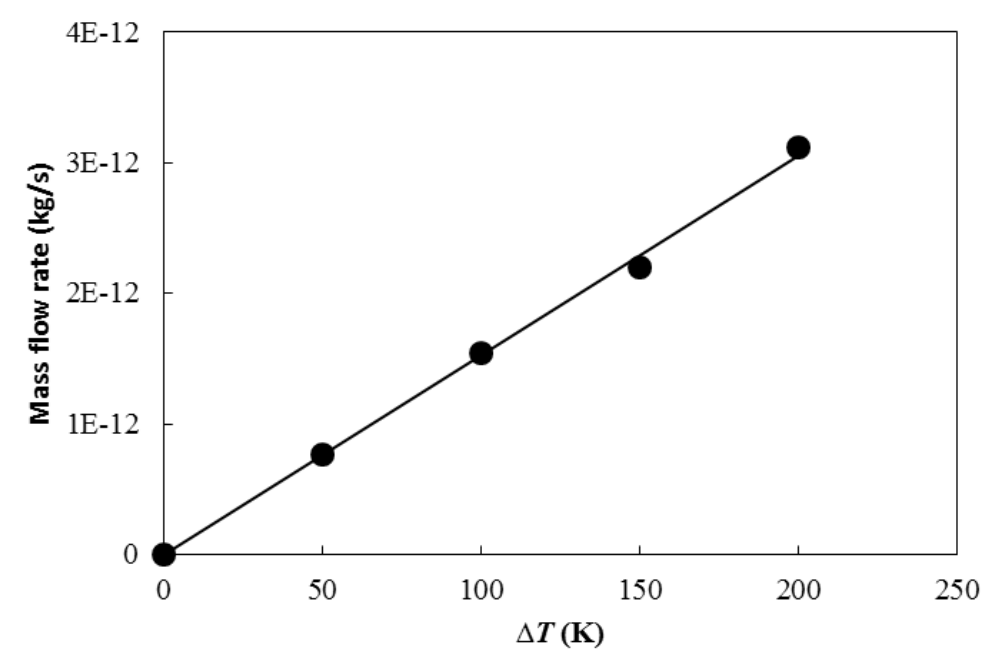

Fig. 5: Mass flow rate for different temperature differences

\subsection{Influence of the Knudsen number}

The influence of the Knudsen number is investigated by considering different initial densities. Figure 6 shows the mass flow rate obtained by DSMC (red points) versus Knudsen 
number, compared with the results from the Navier-Stokes equations associated with firstorder slip boundary condition (black triangles). A maximal value of the mass flow rate obtained by DSMC is reached at a Knudsen number of about 0.09 but the CFD method fails to predict the decrease of the mass flow rate and capture this maximal value. For larger Knudsen numbers, the mass flow rate decreases rapidly and is very low for $K n>1$. It is found that the mass flow rates are negative in the Knudsen number range $[1.2 ; 10.0]$, which indicates that the mean flow in the full transition and early free molecular regimes is in the opposite direction compared to the flow in the slip and early transition flow regimes. A minimal value of the mass flow rate appears at $K n \approx 2.3$. The values of the mass flow rate in the opposite direction are much lower because of a lower mean density reached for larger Knudsen numbers. Nevertheless, the opposite flow is clearly observed by checking the mean velocity across the vertical faces (green dot-dashed line in Fig. 2) where the periodic boundary condition is imposed, as shown in Fig. 7. The maximal mean velocity is found at $K n$ $=0.23$ and the minimal value is observed at $K n=2.3$. The maximal and minimal values of the mass flow rate (Fig. 6) and of the mean velocity (Fig. 7) are not observed for the same values of the Knudsen number, due to the influence of the density which depends both on the local pressure and temperature. 


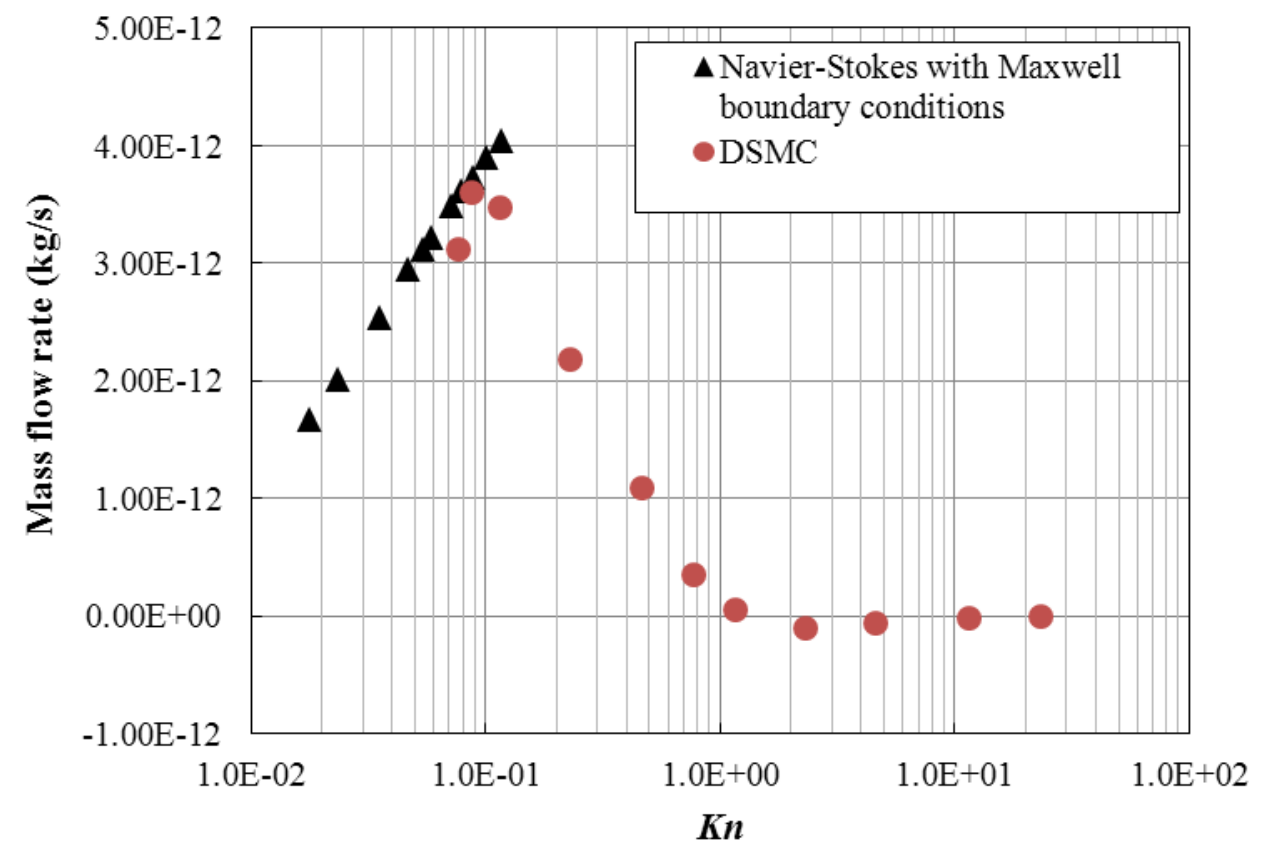

Fig. 6: Mass flow rate versus Knudsen number.

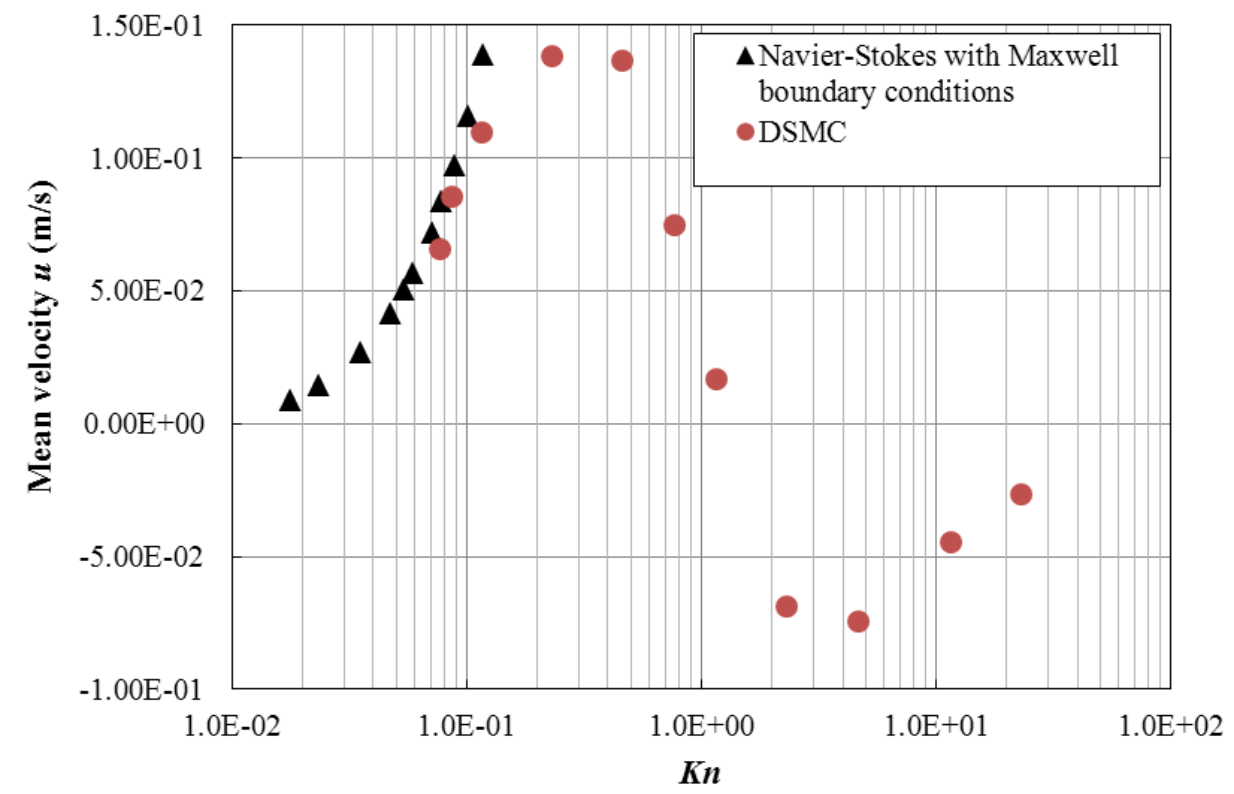

Fig. 7: Mean velocity versus Knudsen number.

Different factors can explain the discrepancies observed between DSMC and CFD 
results. Firstly, the geometric configurations simulated by CFD and DSMC present little differences. As mentioned before, a sharp angle at the intersection of the vertical and inclined segments is considered in the DSMC study, instead of the tip radius introduced in CFD simulations. Several CFD simulations were carried out to estimate the influence of the simplifying assumption which consists in neglecting the radius of curvature at the tip. It was found that the mass flow rate obtained with sharp angles increases of about $15 \%$ to $30 \%$ compared to the case of a radius of curvature $r=50 \mathrm{~nm}$, and that this increase depends on the Knudsen number and temperature difference. As shown in the Section 3.1, the strong tangential temperature gradients appearing around the tips are the principal factors driving the main flow. Therefore, the influence of the shape of the ratchet tips and of their radius of curvature, which are key factors for the design and fabrication of a micropump, will be studied in detail in a future work. Secondly, as mentioned in [14], the results calculated by CFD strongly depend on the coefficients of the velocity slip and temperature jump boundary conditions, namely the velocity slip coefficient, the thermal slip coefficient and the temperature jump coefficient appearing in Eqs. (4) and (5) in [14]. The values of these coefficients reported in the literature may vary in a relative large range, according to the various reported numerical methods [21, 25, 26] and experimental measurements [27]. In Figs. 6 and 7, the classic Maxwell boundary conditions were used for the CFD simulations. These equations and the way in which they have been implemented in a CFD model are detailed in [9]. The viscosity varies with temperature according to the Hard Sphere model.

In Fig. 8, the streamlines and the iso-contours of temperature magnitude are displayed for several representative cases corresponding to $K n=0.23,0.77,1.16$ and 2.32 . It can be seen that the isotherms have a complex distribution, due to the ratchet geometry and the low conductivity of the gas. The tangential temperature gradient near the structured walls is evidenced. On the one hand, the higher the value of the Knudsen number is, the more strongly 
the temperature of the gas near the walls deviates from the wall temperature and the smaller the temperature variations within the gas are. On the other hand, for larger Knudsen numbers, the temperature field near the inclined walls around the tips is more homogenous and is closer to the average temperature as shown in Fig. 8d, while the obviously large temperature gradient along the vertical walls is kept. It probably reduces the effect of the inclined walls in the competition between the flows generated by inclined walls and vertical walls, which results in a vortices growth as the Knudsen number increases. Figure 8 shows totally different flow patterns at $K n=0.23$ and $K n=2.32$. Two main counter-rotating vortices appear in the corners of the ratchets and maintain a flow in the $x$-direction in the center of the channel for $K n=0.23$ and a secondary vortex appears along the lower wall. Up to $K n=1.16$, the main flow is still a rather simple wavy flow going from left to right along a line which curvature changes with $K n$ due to the increasing place occupied by the vortices when $K n$ increases: the upper anticlockwise vortex is stretched by the main flow and the two lower vortices become subparts of a double clockwise vortex. At $K n=2.32$, the vortices are so stretched that they block the main flow in the $x$-direction, and this flow bifurcates, leading to a more complex serpentine flow in the opposite direction, from right to left, as illustrated by the white arrow in Fig. 8d. In the low Knudsen number case (Fig. 8a), the transpiration flow along the vertical walls generates vortices which drag the net flow from left to right; in contrast, for higher Knudsen number (Fig. 8d), the net flow is directly driven in the opposite direction by the transpiration flow along the vertical walls.
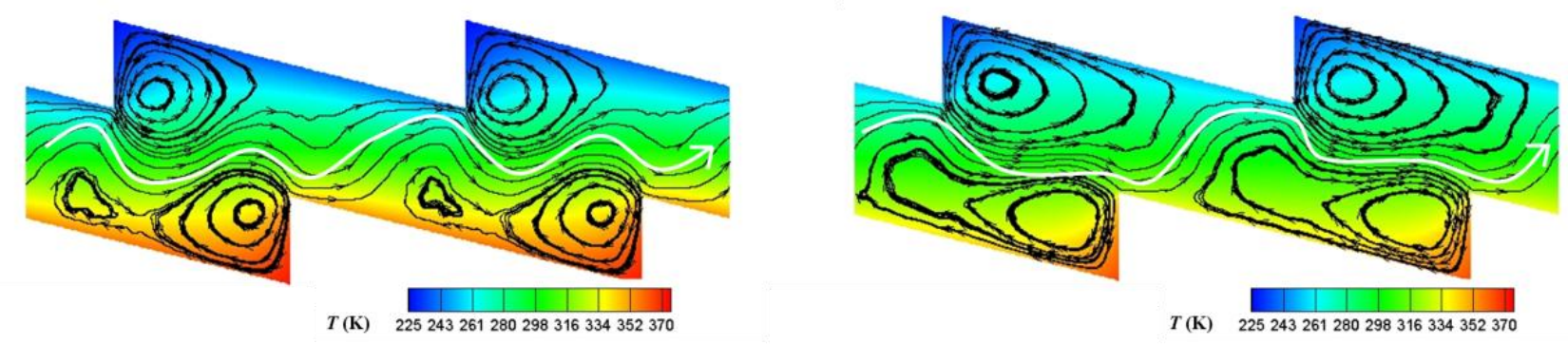
(a)

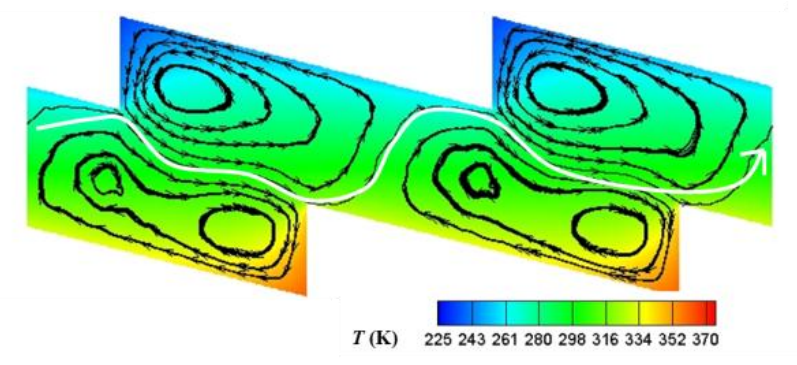

(c) (b)

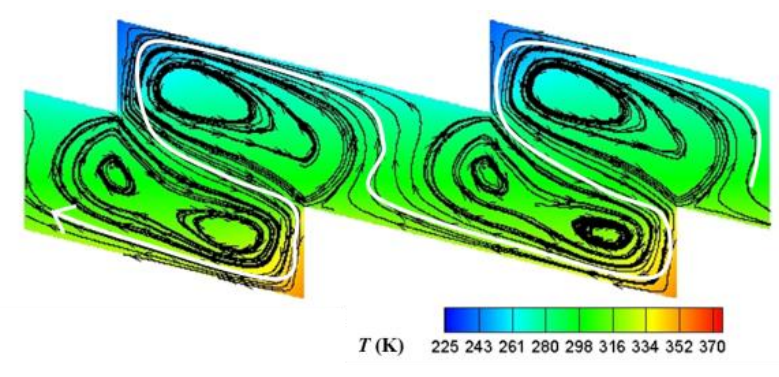

(d)

Fig. 8: Streamlines and iso-contours of temperature for different values of the

Knudsen number: a) $K n=0.23$, b) $K n=0.77$, c) $K n=1.16$ and d) $K n=2.32$

\subsection{Influence of the accommodation coefficients}

In order to separately analyze the effects of the vertical and inclined walls, different accommodation coefficients on the vertical walls and inclined walls are now considered. The temperature contours and the streamlines are shown in Fig. 9 for various values of the accommodation coefficients $\alpha_{V}$ and $\alpha_{I}$. For a quantitative analysis, the calculated mass flow rates are listed in Tables 1 and 2. The competition between the flows generated by the vertical and the inclined walls is clearly shown by the streamlines in Fig. 9. When the accommodation coefficient $\alpha_{V}$ decreases (Figs. 9a to 9c), which corresponds to a smaller effect of the vertical walls, the two main vortices are reduced and more fluid can flow between them. When the inclined walls are diffusive $\left(\alpha_{I}=1\right)$, whatever the value of the accommodation coefficients $\alpha_{V}$ on the vertical walls, the flows is generated in the positive $x$-direction. Conversely, when $\alpha_{I}$ is reduced, two additional vortices are generated near the tips of the ratchet (see Fig. 9d for which $\alpha_{I}=0.9$ to be compared with the reference case of Fig. $8 \mathrm{a}$ for which $\alpha_{I}=1$ ). The two counter-rotating vortices in the neighborhood of each tip laminate the fluid along the vertical walls, generating a negative net flow rate. The vortices disappear when the 
accommodation coefficient $\alpha_{I}$ is reduced to 0.8 (Fig. 9e) and a flow with a larger mass flow rate, whose magnitude increases as $\alpha_{I}$ decreases, is pumped from right to left. The extreme cases are shown in Fig. 9c and Fig. 9f, corresponding to a specular reflection on the vertical walls or on the inclined walls, respectively. Comparing the flows in Fig. 9, it can be seen that the surfaces with the smaller accommodation coefficients have a weaker effect in the competition between the flows generated by the inclined walls and the vertical walls. In the extreme cases, smooth flows are generated either by the vertical walls (Fig. 9f) or by the inclined walls (Fig. 9c), but in opposite directions. However, the flow observed in Fig. 9f ( $\left.\alpha_{V}=1 ; \alpha_{I}=0\right)$, generated by the vertical walls, is much stronger than the flow generated by the inclined walls in Fig. $9 \mathrm{c}\left(\alpha_{V}=0 ; \alpha_{I}=1\right)$, as shown in Tables 1 and 2 . It can be concluded that the flow patterns and the mass flow rates are very sensitive to the local accommodation coefficients. 


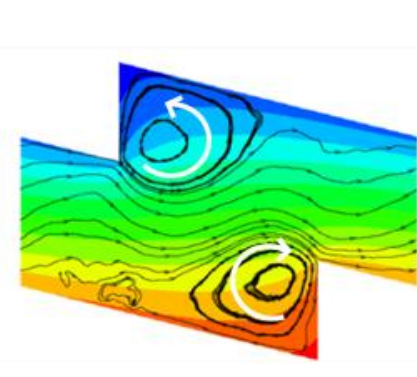

(a)

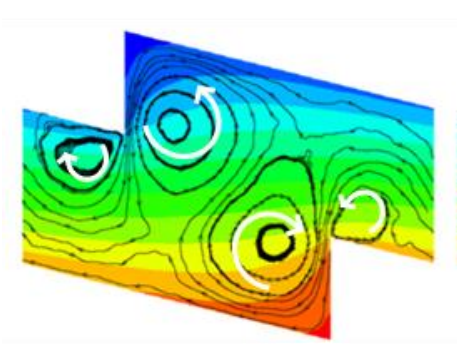

(d)

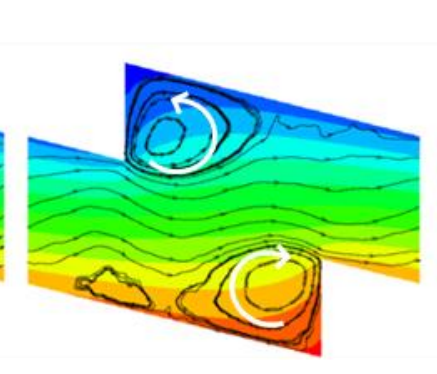

(b)

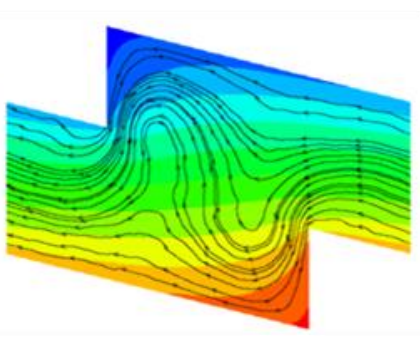

(e)

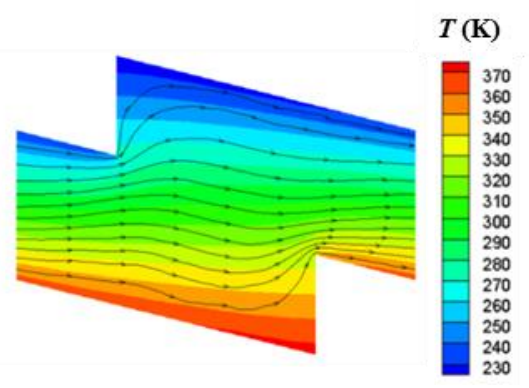

(c)

Fig. 9: Temperature contours and streamlines for $K n=0.23$ and different values of the accommodation coefficients: a) $\alpha_{V}=0.9, \alpha_{I}=1$; b) $\alpha_{V}=0.8, \alpha_{I}=1$; c) $\alpha_{V}=0, \alpha_{I}=1$; d) $\alpha_{V}=1, \alpha_{I}=0.9$; e)

$$
\alpha_{V}=1, \alpha_{I}=0.8 ; \text { f) } \alpha_{V}=1, \alpha_{I}=0 \text {. }
$$

Table 1: Mass flow rate $\left[\times 10^{-12} \mathrm{~kg} / \mathrm{s}\right]$ for different values of the accommodation coefficient $\alpha_{V}$ and full accommodation on the inclined walls $\left(\alpha_{I}=1\right)$.

\begin{tabular}{ccccc}
\hline & $\alpha_{V}=1$ & $\alpha_{V}=0.9$ & $\alpha_{V}=0.8$ & $\alpha_{V}=0$ \\
\hline$\alpha_{I}=1$ & $\mathbf{2 . 1 8}$ & 3.77 & 4.90 & 19.43 \\
\hline
\end{tabular}

Table 2: Mass flow rate $\left[\times 10^{-12} \mathrm{~kg} / \mathrm{s}\right]$ for different values of the accommodation coefficient $\alpha_{I}$ and full accommodation on the vertical walls $\left(\alpha_{V}=1\right)$. 


\begin{tabular}{ccccc}
\hline & $\alpha_{I}=1$ & $\alpha_{I}=0.9$ & $\alpha_{I}=0.8$ & $\alpha_{I}=0$ \\
\hline$\alpha_{V}=1$ & $\mathbf{2 . 1 8}$ & -1.74 & -6.08 & -109.8 \\
\hline
\end{tabular}

\subsection{Influence of geometric parameters}

\subsubsection{Misalignment distance}

The influence of the misalignment distance $L_{m}$ is now studied. The height $d$ of the ratchet and the gap $h$ between the tips are kept constant but the minimum vertical distance $h_{0}$ between the two inclined walls varies with $L_{m}$, according to Eq. (1). Figure 10 presents the variation of the mass flow rate with the misalignment distance $L_{m}$ and the corresponding data are provided in Table 3. It is observed that the worst pumping result is obtained for $L_{m}=1 \mu \mathrm{m}$ , i.e. for $L_{m} / L=0.25$, and that the strongest effect is obtained for $L_{m}$ close to 3, i.e. for $L_{m} / L$ in the range [0.6 - 0.9]. The curve in Fig. 10 is not symmetric due to the asymmetry of the ratchet, which induces a flow with a preferential direction. Actually, $L_{m}$ and $h_{0}$ can be considered as the characteristic lengths controlling the thermal creep flow generated by the vertical walls and by the inclined walls, respectively. On the one hand, for small values of $L_{m}$, the flow generated by the vertical walls is strong and vortices are stretched in the vertical direction, which strongly reduces the net flow rate in $x$-direction, as shown in Figs. 11a and 11b. On the other hand, for higher values of $L_{m}$, the vortices are more compact in the $y$ direction and the flow generated in the $x$-direction is higher, as illustrated by Figs. 11c and 11d. It is found that the maximal reached mass flow rate is 11 times larger than the minimal one and that this dramatic increase is obtained by a simple translation of $2.5 \mu \mathrm{m}$ of one of the surfaces and only requires a heat flux increase of $1 \%$. In other words, controlling the relative position of one wall vis-à-vis the facing wall could be a smart way for a fine tuning of the 
pumping level.

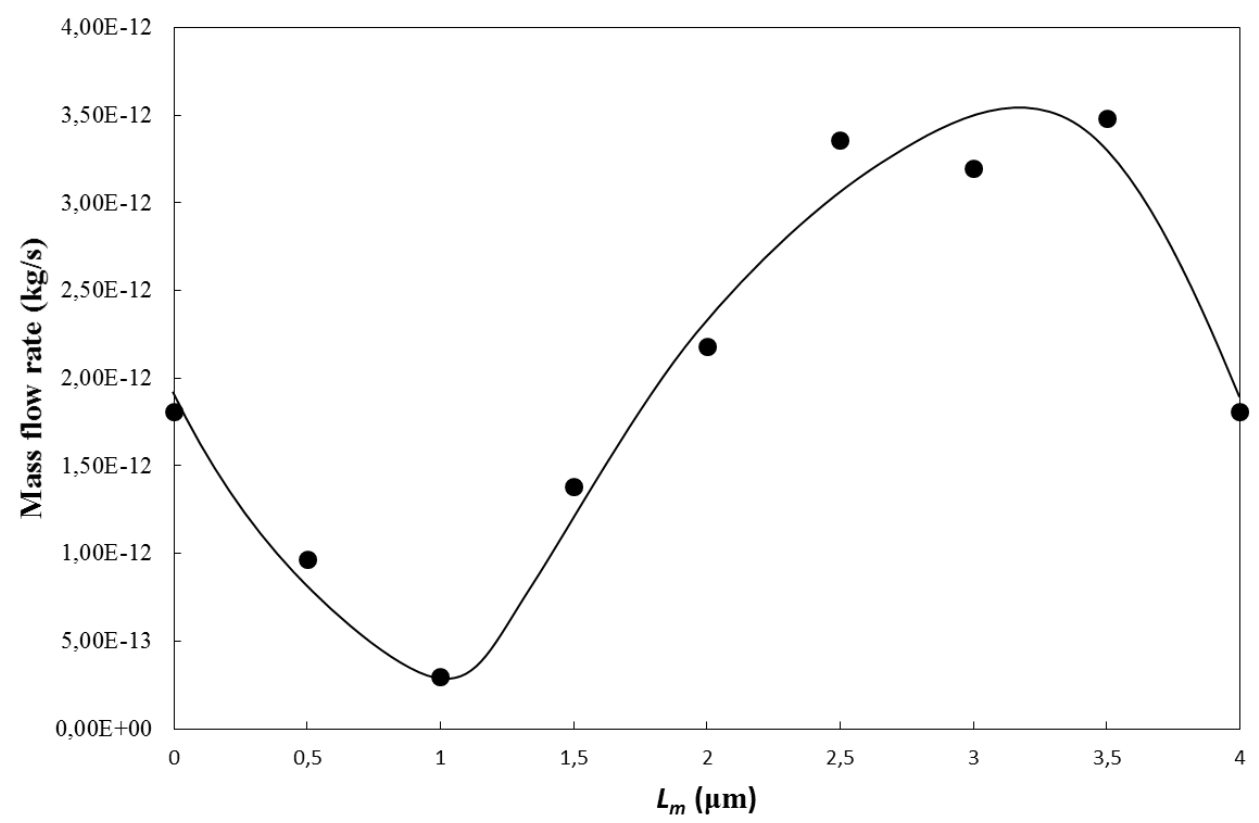

Fig.10: Mass flow rate versus misalignment distance $L_{m}$. $K n=0.23, L=1 \mu \mathrm{m}$ and $d=h=1 \mu \mathrm{m}$

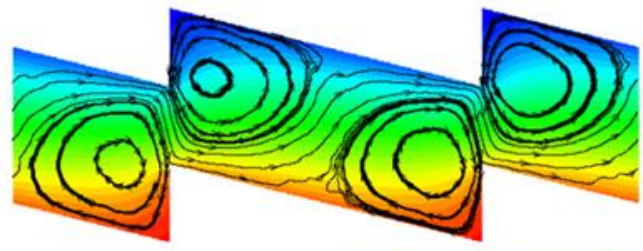

$T(\mathrm{~K})$

$\underset{230250270290310330350370}{D}$ (a)

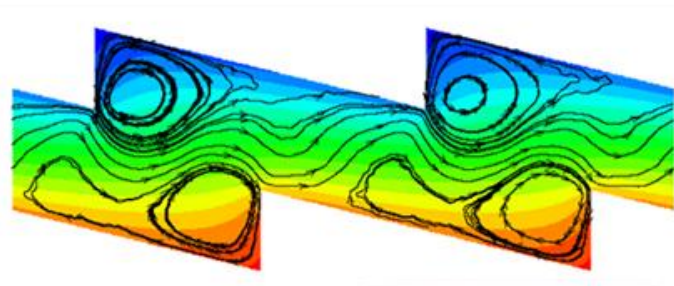

$T(\mathrm{~K})$
230250270290310330350370

(c)

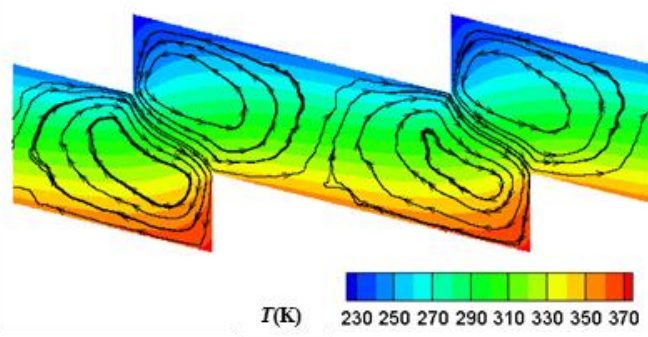

(b)

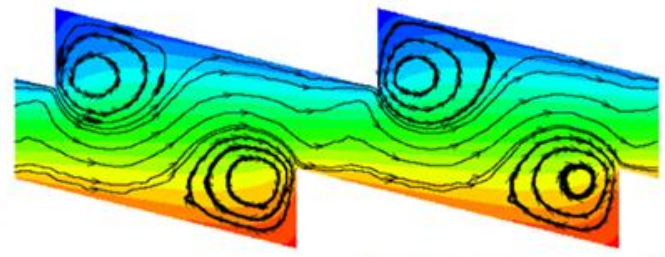

$T(\mathrm{~K})$

230250270290310330350370 
Fig.11: Temperature iso-contours and streamlines for different misalignment distances. $K n=0.23$, $L=1 \mu \mathrm{m}$ and $d=h=1 \mu \mathrm{m}$.

a) $L_{m}=0$; b) $L_{m}=1 \mu \mathrm{m}$; c) $L_{m}=2 \mu \mathrm{m}$; d) $L_{m}=3 \mu \mathrm{m}$

Table 3: Mass flow rate $\left(\times 10^{-12} \mathrm{~kg} / \mathrm{s}\right)$ for different misalignment distances

\begin{tabular}{cccccccccc}
\hline$L_{m}(\mu \mathrm{m})$ & 0 & 0.5 & 1 & 1.5 & 2 & 2.5 & 3 & 3.5 & 4 \\
\hline$h_{0}(\mu \mathrm{m})$ & 1 & 1.875 & 1.75 & 1.625 & 1.5 & 1.375 & 1.25 & 1.125 & 1 \\
Mass flow rate & 1.81 & 0.97 & 0.30 & 1.38 & 2.18 & 3.38 & 3.20 & 3.48 & 1.81 \\
\hline
\end{tabular}

\subsubsection{Ratchet angle}

The influence of the ratchet angle is studied by varying the pattern length $L$, while keeping all other parameters at the reference conditions. Simulations are performed with a pattern length $L$ varying from 1.0 to $6.0 \mu \mathrm{m}$, which corresponds to an angle between $38.7^{\circ}$ and $80.5^{\circ}$. Figure 11 presents the mass flow rate obtained for different ratchet angles in this range and Table 4 provides the precise data. An optimal angle corresponding to a maximal flow rate is found close to $55^{\circ}$, which is in good agreement with the optimal angle of $60^{\circ}$ reported in [14] for a similar geometry but with a $50 \mathrm{~nm}$ radius of curvature for the tips. The decrease of the flow rate is significant when the angle strays from its optimal value. 


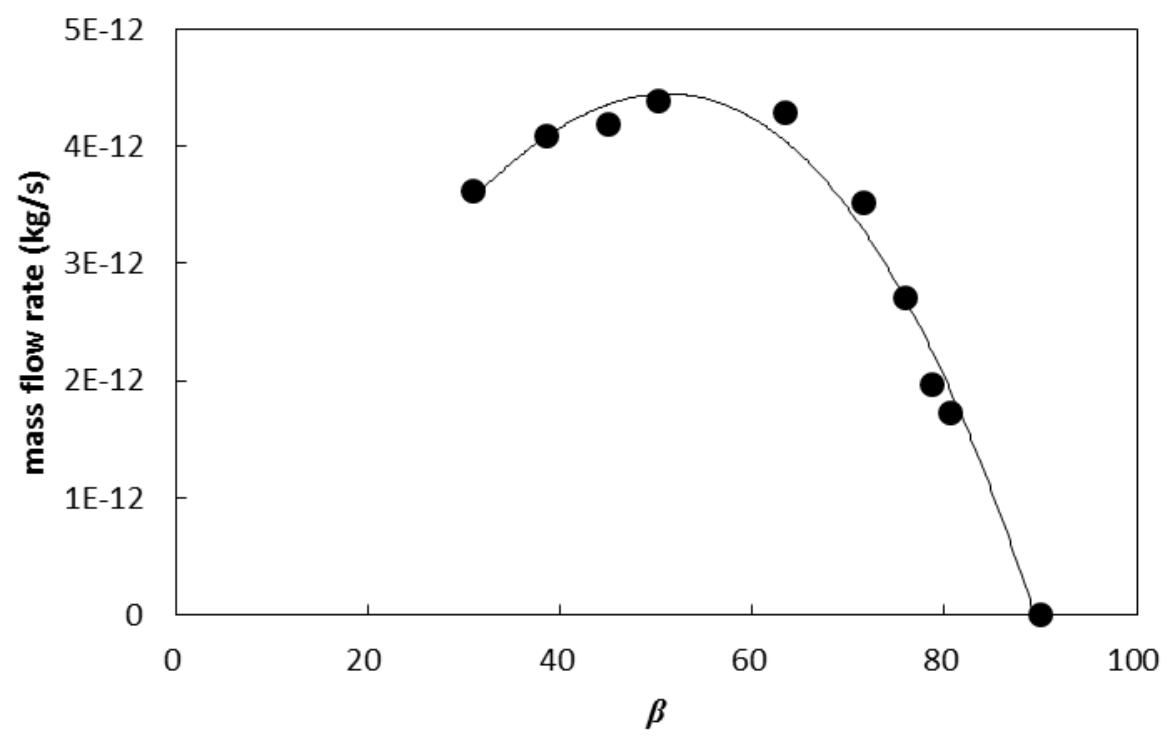

Fig. 12: Mass flow rates for different values of the ratchet angle $\beta$.

$$
K n=0.23, L=1 \mu \mathrm{m} \text { and } d=h=1 \mu \mathrm{m}
$$

Table 4: Mass flow rate $\left(\times 10^{-12} \mathrm{~kg} / \mathrm{s}\right)$ for different values of the ratchet angle $\beta$

\begin{tabular}{cllllllllll}
\hline$L(\mu \mathrm{m})$ & 0.6 & 0.8 & 1.0 & 1.2 & 1.6 & 2.0 & 3.0 & 4.0 & 5.0 & 6.0 \\
$\beta\left({ }^{\circ}\right)$ & 31.0 & 38.7 & 45.0 & 50.2 & 58.0 & 63.4 & 71.6 & 76.0 & 78.7 & 80.5 \\
Mass flow rate & 3.62 & 4.09 & 4.18 & 4.39 & 4.29 & 3.52 & 2.70 & 2.18 & 1.97 & 1.73 \\
\hline
\end{tabular}

\section{Conclusions}

The performance of a new type of Knudsen pump, in which the temperature gradients along the surfaces are simply implemented by means of isothermal cold and hot walls, has been investigated by DSMC simulations. The following conclusions have been observed:

- For the studied configuration, a maximal mass flow rate is reached for a Knudsen number close to 0.09 and for higher rarefaction, the flow rate decreases rapidly as the Knudsen number increases. A net flow is generated in the opposite direction for $K n \in\left[10^{0} ; 10^{1}\right]$.

- The ratchet pump with the present design works as well when all surfaces are diffusive and the mass flow rate is very sensitive to the values of the accommodation coefficients, 
especially the accommodation coefficient of the inclined surfaces.

- The net flow rate and the local flow pattern also depend on the geometric parameters such as the ratchet angle and the misalignment distance. The misalignment of the upper and lower ratchet patterns has a dramatic effect on the resulting flow rate.

However, all simulations presented here are 2D and do not take into account the effect of the lateral walls in the third dimension. In practice, the materials of the lateral walls and the assembly technologies have to be carefully considered in order to avoid a large conductive heat flux between the two micro-structured surfaces through the lateral walls. Additional 3D simulations taking into account conjugate heat transfer will be necessary to estimate the performance limitations of actual ratchet pump designs.

\section{Conflict of interest}

None declared.

\section{Acknowledgements}

The authors acknowledge INSA (Institut National des Sciences Appliquées de Toulouse) which has supported a 3-month stay of S. Stefanov at ICA (Institut Clément Ader). This work was performed using HPC resources from CALMIP (Grant 2015-P1507). The first author acknowledges CSC (China Scholarship Council) which supports her study in France. The second author would like to acknowledge also the financial support provided by the NSF of Bulgaria under Grant No. DUNK 1/3-2009.

\section{References}

[1] M. Knudsen, Eine Revision der Gleichgewichtsbedingung der Gase. Thermische Molekularströmung, Annalen der Physik, 336(1) (1909) 205-229.

[2] N.K. Gupta , S. An, Y.B. Gianchandani, A Si-micromachined 48-stage Knudsen pump for on-chip vacuum, Journal of Micromechanics and Microengineering, 22(10) (2012) 105026. 
[3] R.M. Young, Analysis of a micromachine based vacuum pump on a chip actuated by the thermal transpiration effect, Journal of Vacuum Science \&amp; Technology B, 17(2) (1999) 280-287.

[4] S. McNamara, Y.B. Gianchandani, On-Chip Vacuum Generated by a Micromachined Knudsen Pump, Microelectromechanical Systems, Journal of, 14(4) (2005) 741-746.

[5] Y.-L. Han, E. Phillip Muntz, A. Alexeenko, M. Young, Experimental and Computational Studies of Temperature Gradient-Driven Molecular Transport in Gas Flows through Nano/Microscale Channels, Nanoscale and Microscale Thermophysical Engineering, 11(1-2) (2007) 151-175.

[6] N.K. Gupta, Y.B. Gianchandani, Porous ceramics for multistage Knudsen micropumpsmodeling approach and experimental evaluation, Journal of Micromechanics and Microengineering, 21(9) (2011) 095029.

[7] K. Aoki, P. Degond, L. Mieussens, Numerical simulations of rarefied gases in curved channels: thermal creep, circulating flow, and pumping effect., Communications in Computational Physics 6(5) (2009) 911-954.

[8] K. Aoki, P. Degond, L. Mieussens, S. Takata, H. Yoshida, A Diffusion Model for Rarefied Flows in Curved Channels, Multiscale Modeling \& Simulation, 6(4) (2008) 1281-1316.

[9] V. Leontidis, J. Chen, L. Baldas, S. Colin, Numerical design of a Knudsen pump with curved channels operating in the slip flow regime, Heat Mass Transfer, (2014) 1-16.

[10] D.M. Bond, V. Wheatley, M. Goldsworthy, Numerical investigation into the performance of alternative Knudsen pump designs, Int. J. Heat Mass Transf., 93 (2016) 1038-1058.

[11] H. Sugimoto, Y. Sone, Vacuum pump without a moving part driven by thermal edge flow, in: Rarefied Gas Dynamics: 24th International Symposium on Rarefied Gas Dynamics, AIP Publishing, 2005, pp. 168-173.

[12] A.A. Donkov, S. Tiwari, T. Liang, S. Hardt, A. Klar, W. Ye, Momentum and mass fluxes in a gas confined between periodically structured surfaces at different temperatures, Physical Review E, 84(1) (2011) 016304.

[13] A. Würger, Leidenfrost Gas Ratchets Driven by Thermal Creep, Physical Review Letters, 107(16) (2011) 164502.

[14] J. Chen, L. Baldas, S. Colin, Numerical study of thermal creep flow between two ratchet surfaces, Vacuum, 109(0) (2014) 294-301.

[15] W.-M. Zhang, G. Meng, X. Wei, A review on slip models for gas microflows, Microfluid Nanofluid, 13(6) (2012) 845-882.

[16] G. Bird, Monte Carlo simulation of gas flows, Annual Review of Fluid Mechanics, 10 (1978) 11-31.

[17] G.A. Bird, Molecular gas dynamics and the direct simulation of gas flows, Clarendon Press Oxford, 1994.

[18] M. Vargas, G. Tatsios, D. Valougeorgis, S. Stefanov, Rarefied gas flow in a rectangular enclosure induced by non-isothermal walls, Physics of Fluids (1994-present), 26(5) (2014) 057101.

[19] N.K. Kulakarni, K. Shterev, S.K. Stefanov, Effects of finite distance between a pair of 
opposite transversal dimensions in microchannel configurations: DSMC analysis in transitional regime, Int. J. Heat Mass Transf., 85 (2015) 568-576.

[20] S. Stefanov, R. Barber, M. Ota, D. Emerson, Comparison between Navier-Stokes and DSMC calculations for low Reynolds number slip flow past a confined microsphere, DTIC Document, 2005.

[21] F. Sharipov, Data on the Velocity Slip and Temperature Jump on a Gas-Solid Interface, Journal of Physical and Chemical Reference Data, 40(2) (2011) 023101.

[22] M. Bergoglio, D. Mari, J. Chen, H. Si Hadj Mohand, S. Colin, C. Barrot, Experimental and computational study of gas flow delivered by a rectangular microchannels leak, Measurement, 73 (2015) 551-562.

[23] M. Rojas-Cardenas, I. Graur, P. Perrier, J.G. Meolans, Thermal transpiration flow: A circular cross-section microtube submitted to a temperature gradient, Physics of Fluids (1994present), 23(3) (2011) 031702.

[24] M. Rojas-Cárdenas, I. Graur, P. Perrier, J.G. Méolans, Time-dependent experimental analysis of a thermal transpiration rarefied gas flow, Physics of Fluids (1994-present), 25(7) (2013) 072001.

[25] F. Sharipov, Application of the Cercignani-Lampis scattering kernel to calculations of rarefied gas flows. II. Slip and jump coefficients, European Journal of Mechanics - B/Fluids, 22(2) (2003) 133-143.

[26] J.B. Young, Calculation of Knudsen layers and jump conditions using the linearised G13 and R13 moment methods, Int. J. Heat Mass Transf., 54(13-14) (2011) 2902-2912.

[27] M. Rojas-Cárdenas, I. Graur, P. Perrier, J.G. Méolans, A new method to measure the thermal slip coefficient, Int. J. Heat Mass Transf., 88 (2015) 766-774. 